\title{
EVALUATION OF TIME TO PERFORM SURGERY IN PATIENTS WITH BREAST CANCER ASSISTED AT HOSPITAL FEDERAL DA LAGOA (HFL), IN RIO DE JANEIRO, FROM JANUARY 2014 TO JANUARY 2017
}

Erica Motroni de Almeida', Rafael Henrique Szymanski Machado

${ }^{1}$ Hospital Federal da Lagoa - Rio de Janeiro (RJ), Brazil.

Objectives: The objective of this study was to assess the period of time elapsed between the date of biopsy and the date of surgery of patients with breast cancer (BC) assisted at the Mastology Service of HFL, from January 2014 to January 2017. We excluded from this analysis those patients with distant metastasis and the ones submitted to neoadjuvant chemotherapy. Introduction: Initial studies about the consequences on prognosis of the delay in diagnosis and treatment of $\mathrm{BC}$ tend to show that the longer the delay, the higher the disease staging at diagnosis; which, consequently, leads to lower survival rates. Methods: Retrospective study based on the analysis of medical records. We calculated the time elapsed between the date of biopsy of the malignant lesion and the date of the oncological surgery. The patients were divided in 3 groups regarding the time elapsed between biopsy and surgery: $<60$ days, 60 to 90 days and $>90$ days. Results: The mean waiting time for surgery was of 225.49 days. Only 2 patients (1.80\%) waited less than 60 days. Seven patients (6.31\%) were operated between 61 and 90 days, and the great majority of patients (102, in absolute numbers), waited for more than 90 days (90.89\%). Discussion: Most studies associate the delay in diagnosis and BC treatment with lower survival rates. In a multivariate analysis, major delays to start the treatment were a significant risk factor for the reduction in survival. The delay in the surgical treatment of younger women (number of weeks between the date of diagnosis and date of definitive treatment) was assessed in a retrospective, case-control study published in 2013, which used data from the California Cancer Registry Database. In this study, the five-year survival of women treated with surgery who waited more than 6 weeks was $80 \%$, in comparison to $90 \%$ among those whose delay was shorter than 2 weeks. Another retrospective study assessed the impact of the delay of the beginning of treatment after the biopsy confirmed BC. This analysis showed that the delay to begin the first treatment longer than or equal to 60 days was associated with worse specific survival rates. Gagliato et al showed the impact of the delay to start adjuvant chemotherapy in patients with BC in several stages, and with different tumor subtypes. The results showed worsened survival rates when the beginning of adjuvant chemotherapy was delayed in all of the study groups. Conclusion: Considering the data in this study and data from several others regarding the negative impact of delay in $\mathrm{BC}$ treatment, it is clear that efforts in all spheres of the government should be made so that the healing and survival rates can improve. 